\title{
Vascular Ehlers-Danlos syndrome with distinct histopathologic features
}

\author{
Hee Sang Hwang ${ }^{1}$, Jin Woo Song ${ }^{2}$, Se Jin Jang ${ }^{1}$ \\ Departments of ${ }^{1}$ Pathology and ${ }^{2}$ Internal Medicine, Asan Medical Center, University of Ulsan College of Medicine, Seoul, Korea
}

Ehlers-Danlos syndrome is a connective tissue disorders that presents with heterogeneous manifestations depending on the involved genes. Vascular Ehlers-Danlos syndrome (vEDS), also known as Ehlers-Danlos syndrome type IV, is caused by a heterozygous mutation in the COL3A1 gene that encodes the pro- $\alpha 1$ chains of type III collagen, which attenuates the structural integrity of type III collagen-enriched tissues, such as skin, lung, liver, intestine, and blood vessel. vEDS can result in fatal complications such as arterial or intestinal rupture, but its clinical diagnosis is often challenging. Here, we present a case of vEDS that was diagnosed based on histopathologic and confirmatory genetic examinations.

\section{CASE REPORT}

A 19-year-old male was admitted to our hospital for recurrent episodes of spontaneous pneumothorax (6 times), which started about 8 months ago. His father had a history of recurrent pneumothorax and also suffered from spontaneous intraabdominal bleeding due to aneurysmal rupture of the common hepatic artery 7 years ago. The patient had undergone wedge resection of the lung at another hospital, after which he experienced a secondary pneumothorax, but no specific pathologic diagnosis was made. Thoracic computed tomography at our hospital showed multiple parenchymal cystic lesions with surrounding ground-glass opacity and several small nodular lesions in both lungs (Fig. 1A), suggesting vasculitis with hemorrhage, infected cystic lung disease, or paragonimiasis. However, neither serum

Received: December 27, 2020 Revised: February 23, 2021 Accepted: March 23, 2021

Corresponding Author: Hee Sang Hwang, MD, PhD

Department of Pathology, Asan Medical Center, University of Ulsan College of Medicine, 88 Olympic-ro 43-gil, Songpa-gu, Seoul 05505, Korea Tel: +82-2-3010-1869, Fax: +82-2-472-7898, E-mail: hshwang0908@amc.seoul.kr anti-nuclear antibody nor anti-Paragonimus Westermani antibody titer was elevated. No abnormality in the thoracic greater vessels was identified. Abnormalities of the extrathoracic organs were not evaluated at the time of the admission. Pathological examination of the wedge-resected lung specimen slides sent from an external pathology laboratory revealed intra-alveolar accumulation of red blood cells and hemosiderin-laden macrophages (Fig. 1B, C), indicating recurrent episodes of intra-alveolar hemorrhage. The most diagnostic histologic finding was the presence of several round fibro-osseous nodules scattered in a hemorrhagic background of lung parenchyma (Fig. 1D, E). Disruption of the vascular wall was not identified, even in the examination under van Gieson elastic staining. Due to suspicion of vEDS based on the patient's familial history and the histopathologic findings, COL3A1 gene sequencing was performed. The sequencing test revealed a heterozygous germline mutation in the spliceosome acceptor site in intron 23 of the COL3A1 gene (COL3A1 c. $1662+1 \mathrm{G}$ > A [p. Gly537_Pro554del]) (Fig. 1F), a known pathogenic mutation in the ClinVar database (https://www.ncbi.nlm.nih.gov/clinvar/ variation/101269/). Hence, the patient was diagnosed with vEDS.

\section{DISCUSSION}

vEDS comprises about 4\%-5\% of Ehlers-Danlos syndrome cases and has a high rate of fatal complications related to cardiovascular and gastrointestinal systems, such as cerebrovascular hemorrhage or intestinal rupture. In addition, pregnancy can be fatal in patients with vEDS who might develop ruptures in the uterus or uterine vessels [1]. Therefore, prompt diagnosis is the mainstay of medical management of vEDS to lower the rate of such fatal complications [2]. However, the rarity of vEDS makes its diagnosis challenging, frequently leading to delayed diagnosis. The pathologic diagnosis of vEDS is challenging because the characteristic histopathological findings of vEDS are not widely 

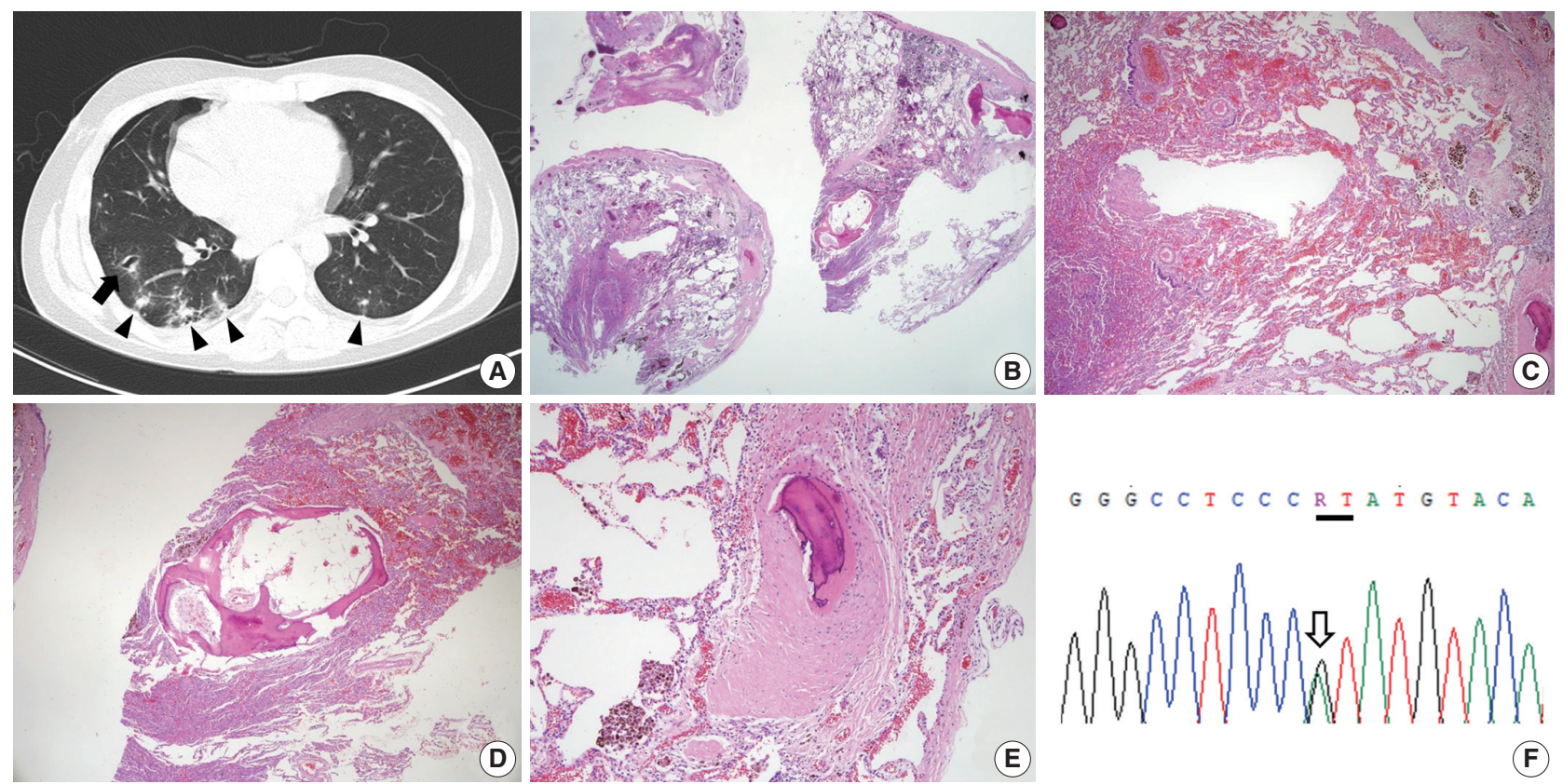

Fig. 1. Radiological and pathological features of the present case. (A) Chest computed tomography, performed after the patient's second episode of spontaneous pneumothorax, showing a parenchymal cystic lesion with an irregularly thickened cavity wall and surrounding ground-glass opacity (arrow). Several ill-defined nodular opacities with adjacent ground-glass opacities, predominant in the basal lungs, were identified (arrowheads). (B) Photomicrogram of the surgical lung biopsy specimen showing multifocal intra-alveolar accumulations of red blood cells and fibro-osseous nodules. (C) Magnified photomicrogram revealing intra-alveolar collections of red blood cells and hemosiderin-laden macrophages. (D, E) Magnifications of a parenchymal fibro-osseous nodule in the background of parenchymal hemorrhage. (F) Sanger sequencing test for the COL3A1 gene of the patient, showing a heterozygous pathogenic germline mutation (empty arrow) at the spliceosome acceptor site in intron 23 of the COL3A1 gene (black line) (COL3A1 c. 1662 + 1G>A [p. Gly537_Pro554del]).

recognized. Abrupt caliber changes in the muscularis propria with diverticular formation have been identified in colon resection specimens of vEDS patients [3]. Kawabata et al. [4] reported that fragile lung parenchyma with spontaneous laceration, acute hemorrhage and/or hematoma, and fibro-osseous nodules, which are hypothesized to be a consequence of the organization of hematomas, are frequently identified in surgical lung biopsy specimens of vEDS patients, findings which serve as clues for pathologic diagnosis. In line with these findings, a previous report described a case of vEDS that was diagnosed based on identification of fibro-osseous nodules and vascular disruption in the lung parenchyma, suggesting the potential of histopathologic features in clinical diagnosis of vEDS [5]. Therefore, the distinct histopathologic features of vEDS should be considered when assessing surgical lung biopsy specimens from patients with recurrent spontaneous pneumothorax and/or hemorrhagic lung parenchymal cysts.

In conclusion, identifying characteristic histopathologic features in surgical lung biopsy samples, such as parenchymal fragility, spontaneous laceration, parenchymal hemorrhage, and fibroosseous nodules, can contribute to early clinical diagnosis and proper management of vEDS.

\section{Ethics Statement}

This case study was approved by the Institutional Review Board of Asan Medical Center (2020-1889), which waived the requirement for informed consent.

\section{Availability of Data and Material}

Data sharing not applicable to this article as no datasets were generated or analysed during the study.

\section{Code Availability}

Not applicable.

\section{ORCID}

Hee Sang Hwang https://orcid.org/0000-0001-9486-9214 Jin Woo Song https://orcid.org/0000-0001-5121-3522 Se Jin Jang https://orcid.org/0000-0001-8239-4362

\section{Author Contributions}

Conceptualization: SJJ, JWS, HSH. Data curation: HSH. Formal analysis: HSH, SJJ. Investigation: HSH. Methodology: HSH, JWS. Resources: SJJ, JWS. Supervision: SJJ. Writing_original draft: HSH. Writing_review \& editing: HSH, SJJ. Approval of final manuscript: all authors. 


\section{Conflicts of Interest}

The authors declare that they have no potential conflicts of interest.

\section{Funding Statement}

No funding to declare.

\section{References}

1. Pepin M, Schwarze U, Superti-Furga A, Byers PH. Clinical and genetic features of Ehlers-Danlos syndrome type IV, the vascular type. N Engl J Med 2000; 342: 673-80.

2. Shalhub S, Black JH 3rd, Cecchi AC, et al. Molecular diagnosis in vascular Ehlers-Danlos syndrome predicts pattern of arterial in- volveent and outcomes. J Vasc Surg 2014; 60: 160-9.

3. Blaker H, Funke B, Hausser I, Hackert T, Schirmacher P, Autschbach F. Pathology of the large intestine in patients with vascular type Ehlers-Danlos syndrome. Virchows Arch 2007; 450: 713-7.

4. Kawabata Y, Watanabe A, Yamaguchi S, et al. Pleuropulmonary pathology of vascular Ehlers-Danlos syndrome: spontaneous laceration, haematoma and fibrous nodules. Histopathology 2010; 56: 944-50.

5. Berezowska S, Christe A, Bartholdi D, Koch M, von Garnier C. Pulmonary fibrous nodule with ossifications may indicate vascular Ehlers-Danlos syndrome with missense mutation in COL3A1. Am J Respir Crit Care Med 2018; 197: 661-2. 